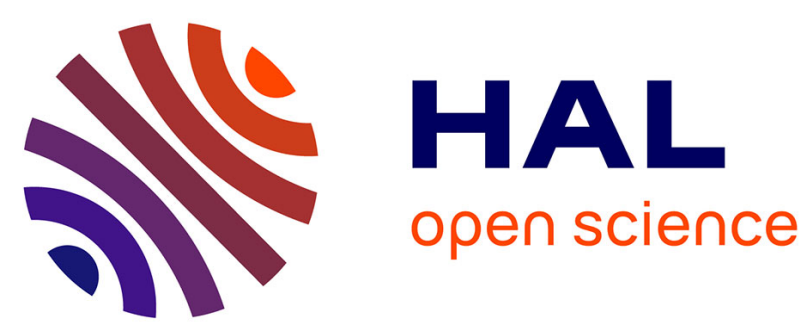

\title{
Randomised Controlled Trial of Screening and Prophylactic Treatment to Prevent Primary Angle Closure Glaucoma.
}

Jennifer Ly Yip, Paul J Foster, Davatseren Uranchimeg, Balt Javzandulam, Dash Javzansuren, Tsengenbayar Munhzaya, Pak S Lee, Jamyanjav

Baassanhuu, Clare E Gilbert, Peng Khaw, et al.

\section{To cite this version:}

Jennifer Ly Yip, Paul J Foster, Davatseren Uranchimeg, Balt Javzandulam, Dash Javzansuren, et al.. Randomised Controlled Trial of Screening and Prophylactic Treatment to Prevent Primary Angle Closure Glaucoma.. British Journal of Ophthalmology, 2010, 94 (11), pp.1472. 10.1136/bjo.2009.168682 . hal-00557334

\section{HAL Id: hal-00557334 https://hal.science/hal-00557334}

Submitted on 19 Jan 2011

HAL is a multi-disciplinary open access archive for the deposit and dissemination of scientific research documents, whether they are published or not. The documents may come from teaching and research institutions in France or abroad, or from public or private research centers.
L'archive ouverte pluridisciplinaire HAL, est destinée au dépôt et à la diffusion de documents scientifiques de niveau recherche, publiés ou non, émanant des établissements d'enseignement et de recherche français ou étrangers, des laboratoires publics ou privés. 


\section{Randomised Controlled Trial of Screening and Prophylactic Treatment to Prevent Primary}

\section{Angle Closure Glaucoma.}

4 Jennifer L.Y.Yip ${ }^{1,2 A, 3}$ MSc MRCOphth MFPH, Paul J.Foster 1,2a, 4 PhD FRCS(Ed), Davatseren

$5 \quad$ Uranchimeg $^{5}$ PhD MD, Balt Javzandulam ${ }^{5}$ MD, Dash Javzansuren ${ }^{5}$ MD, Tsengenbayar Munhzaya ${ }^{5}$

6 MD, Pak .S.Lee ${ }^{2 A}$ MPhil, Jamyanjav .Baassanhuu ${ }^{5}$ PhD MD, Clare.E.Gilbert ${ }^{1}$ MD FRCOphth, 7 Peng.T.Khaw ${ }^{2 B, 4}$ PhD FRCOphth, Gordon J.Johnson ${ }^{1}$ MD FRCOphth, Winifred P. Nolan ${ }^{6}$ MD 8 FRCOphth

${ }^{1}$ International Centre for Eye Health, London School of Hygiene and Tropical Medicine, London, UK

${ }^{2}$ UCL Institute of Ophthalmology, London, UK

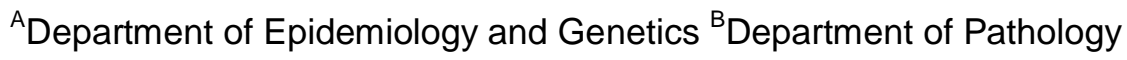

${ }^{3}$ Department of Public Health and Primary Care, Institute of Public Health, Forvie Site, Robinson

Way, University of Cambridge, UK

${ }^{4}$ National Biomedical Research Centre for Ophthalmology, Moorfields Eye Hospital

${ }^{5}$ Department of Ophthalmology, Health Sciences University, Ulaanbaatar, Mongolia and

${ }^{6}$ Birmingham and Midland Eye Centre, Birmingham UK

Financial Support: The Wellcome Trust, British Council for Prevention of Blindness (London),

Christian Blind Mission (CBM) (Bensheim), The National Lotteries Fund through Fight for Sight

(London). The Yag laser used in this study was donated by the Velux Foundation, Copenhagen.

The authors acknowledge a proportion of their financial support from the Department of Health

through the award made by the National Institute for Health Research to Moorfields Eye Hospital

Centre for Ophthalmology. The views expressed in this publication are those of the authors and not necessarily those of the Department of Health.

There was no conflict of interest. 
29 Address for correspondence: Dr Winifred Nolan, Birmingham and Midland Eye Centre, Dudley Road,

30 Birmingham B18 7QU, UK. Email: winnie_nolan@yahoo.com

31 Word count: Abstract:243 Text: 3076 excluding title page, legends and references.

32

33 Licence for Publication

34 "The Corresponding Author has the right to grant on behalf of all authors and does grant on behalf of 35 all authors, an exclusive licence (or non exclusive for government employees) on a worldwide basis

36 to the BMJ Publishing Group Ltd and its Licensees to permit this article (if accepted) to be published

37 in BJO editions and any other BMJ PGL products to exploit all subsidiary rights, as set out in our

38 licence(http://group.bmj.com/products/journals/instructions-for-authors/licence-forms/)." 
Purpose: To determine if screening with ultrasound A-scan and prophylactic treatment of primary angle closure (PAC) with laser peripheral iridotomy (LPI) can reduce the incidence of primary angle closure glaucoma (PACG) in Mongolia.

Methods: A single-masked randomised controlled trial was initiated in 1999. 4725 volunteer Mongolian participants aged $\geq 50$ years old from the capital Ulaanbaator or rural province of Bayanhongor were recruited, of which 128 were excluded with glaucoma. 4597 were randomly allocated to the control, no-screening arm or screening with ultrasound central anterior chamber depth (CACD), with cut off set at $<2.53 \mathrm{~mm}$. 685 screen positive participants were examined and angle-closure was identified by gonioscopy in 160, of which 156 were treated with prophylactic LPI. Primary outcome of incident PACG was determined using both structural and functional evidence from objective grading of paired disc photographs from baseline and follow up, objective grading of follow up visual fields and clinical examination.

Results: Six years later, 801 (17.42\%) participants were known to have died, and 2047 (53.92\%) were traced and underwent full ophthalmic examination. In an intention to treat analysis using available data, PACG was diagnosed in 33 participants $(1.61 \%, 95 \%$ confidence interval $(\mathrm{Cl})=1.11$ $2.25 \%$ ), of which 19 were in the screened group and 14 in the non-screened group (Odds Ratio (OR) $=1.29,95 \% \mathrm{Cl}=0.65-2.60, \mathrm{p}=0.47)$ indicating no difference between groups. screening with $c A C D<2.53 \mathrm{~mm}$ and prophylactic treatment of PAC. 
Primary angle closure glaucoma (PACG) is a major cause of blindness. By 2020, an estimated 5.9 million will be blind from PACG worldwide, of which $60 \%$ will be in East Asia. ${ }^{1}$ Chronic PACG is the more prevalent form of disease; with only a third having a history of an acute episode. ${ }^{2}$ Historical studies have shown that prophylactic peripheral iridotomy can prevent acute angle closure in fellow eyes. ${ }^{3,4}$ More recent studies have also demonstrated that laser iridotomy can stabilise elevated intraocular pressure in non-glaucomatous eyes with occludable angles and primary angle closure (PAC). ${ }^{5}$ Therefore, early detection and treatment can potentially reduce the incidence of PACG. A shallow central anterior chamber depth (CACD) is a strong risk factor for primary angle closure (PAC) in populations with a high prevalence of PACG. ${ }^{6,7}$ Measurement of CACD is effective in detecting occludable angles and PAC when compared with gonioscopy. Occludable angles and PAC therefore fulfil many of the Wilson-Jungner criteria for screening. ${ }^{8}$

We report the primary outcome of a randomised controlled trial of screening and prophylactic treatment of PAC to prevent PACG in Mongolia.

\section{METHODS}

Details of methods at baseline have been published..$^{9}$ The International Society for Geographical and Epidemiological Ophthalmology (ISGEO) criteria $^{10}$ were used for diagnosis of glaucoma and angle closure throughout the study This trial is reported in line with the CONSORT statement.

\section{Baseline (1999):}

Prior to field-work a computer generated allocation sequence was produced in London and stored in sealed envelopes. Mongolian volunteers aged $\geq 50$ years old living in the capital UlaanBaator or the rural province of Bayankhongor for $>3$ months were recruited. After obtaining consent and randomisation, all underwent optic disc assessment to exclude those with glaucomatous optic neuropathy (GON). Optic discs of both eyes in all participants were photographed using a nonmydratic fundus camera (Canon CR4-45NM, Japan). 
Participants randomised to intervention were screened with cACD measurement using a slitlamp mounted ultrasound biometer (Allergan-Humphrey model 820, Allergan, London, UK). Intraocular pressure (IOP) was measured with a Tonopen (Mentor Ophthalmics, MA, USA). A positive screening test was defined as CACD $<2.53 \mathrm{~mm}$ or IOP $\geq 24 \mathrm{mmHg}$. A cut off of $<2.53 \mathrm{~mm}$ produced sensitivity of $77 \%$ and a specificity of $83 \%$ with a positive predictive value of $32 \%$. This method performed better than handheld A-scan and is a relatively cheap and convenient instrument for screening. ${ }^{11} \mathrm{~A}$ Tonopen IOP of $24 \mathrm{mmHg}$ was selected as the mean plus two standard deviations based on a random sample of 769 Mongolians. Participants who screened positive underwent a detailed ophthalmic examination including visual acuity, slitlamp biomicroscopy, Goldmann applanation tonometry (GAT) and dilated fundus examination. Visual fields were tested using a 56 point threshold strategy (Henson 3000, Topcon Medical Instruments, Newbury, UK) on patients with evidence of optic disc characteristics indicating possible GON. The drainage angle was assessed with a two mirror Goldmann lens and graded as open or occludable ${ }^{10}$. Participants with occludable or closed angles were offered bilateral laser iridotomies using a portable Yag laser (Visualas II, Carl Zeiss Ltd, Germany) prior to dilated examination. Participants randomised to the control, non-screening group received no further examination or treatment. Neither participants nor baseline staff were masked to group allocation. The flow of participants is shown in Figure 1. Between baseline and follow up, participants had access to the usual care provided by local services.

\section{Follow up (2005):}

112 Participants were traced using available records from the baseline study. The names of untraceable

113 participants were submitted to the central registration office in Ulaanbaatar to check for deaths and 114 new addresses.

115 All traced participants received a detailed examination, similar to that performed at baseline. One ophthalmologist assessed the presence and patency of a LPI at follow up, whilst gonioscopy and

117 dilated examination of the lens and optic disc was undertaken by a second ophthalmologist, masked 118 to the allocation status of the participant throughout the field work. . 
120 The same instrument and observer measured the CACD on all patients. A dilated examination was

121 also performed on all who consented, to determine the presence of posterior synechiae, lens opacity,

122 and assessment of the optic disc using an eyepiece mounted graticule to record cup disc ratio

123 (CDR). All participants underwent dilated disc photography. Patients with evidence of structural

124 glaucomatous damage or glaucoma suspects ${ }^{10}$ had a visual field assessment. (Table 1) If the visual

125 field was considered abnormal, the participant was advised to return for a repeat assessment.

126 Because of the high numbers of subjects in the trial and the fact that many people travelled long

127 distances to attend the clinic and could not stay for a long time, we were unable to perform visual

128 fields on all participants due to the limited time available. People diagnosed with occludable angles

129 were. provided with one dose of acetazolamide $500 \mathrm{mg}$ and offered laser treatment the following day.

130 All patients diagnosed with glaucoma were treated appropriately with topical medication and

131 referredto the local ophthalmologist.

132 Previous acute angle closure was diagnosed at follow up based on a history of sudden onset of

133 severe pain and blurred vision with evidence of glaucomflecken, iris whorling or the presence of a PI

134 which had not been performed at the baseline study.

135

136 Study Outcomes

137 The primary outcome was incident PACG. This was diagnosed either clinically in the field, or based on optic disc progression with visual field evidence of glaucomatous defect using objective criteria.

139 PACG was diagnosed where there was evidence of both an occludable angle and GON with no 140 evidence of secondary causes of glaucoma.

142 Optic disc progression was determined by side by side comparison of baseline and follow up 143 monoscopic photographs at the Moorfields Image Reading Centre. The disc grading protocol was 144 based on the Ocular Hypertension Treatment Study disc grading protocol, and used the same criteria

145 to determine optic disc progression. ${ }^{12} \mathrm{~A}$ standardised set of photographs was developed by

146 glaucoma specialists and used to illustrate each aspect of disc progression. 
Evidence of glaucomatous visual field defect was determined by two independent masked glaucoma specialists (PJF and WN), masked to the intervention allocation of the participant using standardised criteria. Agreement on visual field status was required for overall grading for each participant by both graders. When disagreement occurred, the visual fields for that participant were reassessed by both

152 graders together and a consensus reached.

Incident PACG was identified by any or a combination of methods above, based on structural and functional changes or structural changes only. Structural changes were identified from clinical examination and/or photographic evidence of optic disc progression.

157

The study was conducted according to the Declaration of Helsinki, and ethical approval was obtained from the Ministry of Health, Ulaanbaator, Mongolia (baseline and follow up) and the London School of Hygiene and Tropical Medicine (follow-up). All participants gave informed consent in the local language.

\section{Statistical Methods}

164 The sample size calculations were based on a 5 year incidence of PACG of $1.4 \%$ (95\% confidence interval $0.68 \%-2.1 \%$ ). An estimated sample size of 5000 participants would have $85 \%$ power to detect a $70 \%$ reduction in risk of incident PACG and allow for $33 \%$ loss to follow up. We followed an intention to treat analysis strategy, where all patients were analysed according to randomisation group. Comparison of PACG incidence was patient based using the $x^{2}$ test.

\section{Missing data}

171 Analyses for primary outcome were performed on observed data only. Due to the limitations of

172 missing data from loss to follow up, we used a multiple imputation procedure ${ }^{13}$ in a sensitivity

173 analysis. The purpose of this part of the analysis was to determine whether the main results were

174 within limits of estimates obtained from imputed data. The multiple imputation procedure substitutes

175 missing values with several versions of imputed values. 50 sets of imputed data were generated. 
All statistical analyses were carried out using Stata 10 (Statcorp, Texas). The multiple imputation

178

179

180

181

182

183

184

185

186

187

188

189

190

191

192

193

194

195

196

197

198

199

200

201

202

203

204

205

procedure and analysis were implemented with multiple imputation using chained equations

commands "ice" and "mim". ${ }^{14,15}$ This procedure is described further in the appendix.

\section{RESULTS}

The baseline study bas previously been described in detail. Of 4,725 volunteers randomised and assessed for eligibility in 1999, 128 were excluded at baseline with glaucoma. Due to the logistical limitations and participant flow in the clinic during the baseline study, glaucoma was excluded after randomisation.

In 2005 , a total 33 of 2,047 examined participants $(1.61 \%, 95 \%$ confidence interval $(\mathrm{Cl})=1.11-2.25 \%)$ were diagnosed with incident PACG, of which $19(1.81 \%, 95 \% \mathrm{Cl}=1.09-2.81 \%)$ were in the screened arm and $14(1.40 \%, 95 \% \mathrm{Cl}=0.77-2.34 \%)$ were in the no screening arm, with no evidence of a statistically significant difference between groups (Odds Ratio $(\mathrm{OR})=1.29,95 \% \mathrm{Cl}=0.65-2.60$, $\mathrm{p}=0.47)($ Table 2). Nineteen patients had both structural and functional evidence of PACG, of which seventeen patients had reproducible field defects. Fourteen patients were diagnosed using structural evidence only. For all types of evidence, there were greater or equal numbers of patients diagnosed with PACG in the screening arm, but there was no evidence of a statistical difference between groups from any diagnostic category. Ten of nineteen participants from the screening arm diagnosed with PACG were screen positive and examined in 1999. Of these, four had been diagnosed with occludable angles (PAC) and treated with laser PI, and six did not have occludable angles. A further nine participants in the screening arm were screen negative.

Forty two participants had been treated between the baseline and follow up studies: Ninteen (1.81\%) were in the screened arm and $23(2.31 \%)$ in the non-screened arm (Table 3). Of the 33 patients diagnosed with incident PACG, seventeen had evidence of a patent iridotomy, of which only four (of 17) had been performed at baseline.

Six cases of acute angle closure (AAC) were elicited based on clinical examination and history at follow up, two of which were in the intervention group, and four in the control group. All were seen to 
have patent peripheral iridotomies that had been performed between 1999 and 2005. The two

207

208

209

210

\section{1}

212

213

214

215

216

217

218

219

220

221

222

223

224

225

226

227

228

229

230

231

232

233

234

patients with previous AAC in the screened group both had baseline CACD $>2.53 \mathrm{~mm}$ and did not undergo a slitlamp examination in 1999; both were diagnosed with PACG at follow up. Of the four patients in the control group with previous AAC, two were diagnosed with PACG and two with PAC.

Death was verified in similar proportions of participants from both arms (17.58\% vs $17.27 \%, p>0.79)$. There was no evidence of localised lens opacity in any participants treated with LPI at baseline. Out of 80 participants with follow up who had LPI at baseline, dilated examination was available for 75 right eyes and 73 left eyes. New posterior synechiae were identified in 4 right eyes and 3 left eyes. Compared to participants who were examined but did not receive LPI, there was no evidence of increased risk of posterior synechiae associated with LPI at baseline $(4 / 75=5.33 \%$ vs $4 / 229=1.75 \%$, $p=0.09$ right eye $3 / 73=4.11 \%$ vs $5 / 229=2.18 \%, p=0.37$ left eye).

A sensitivity analysis with imputed data to include all 4795 participants randomised supported the findings from the complete case analysis. The observed odds ratios fell within the range of $95 \%$ confidence intervals generated from analyses from imputed data (overall 95\% Cl from $0.57-2.20$, all $p>0.7)$

\section{DISCUSSION}

We found that screening with cACD cut off value $<2.53 \mathrm{~mm}$ and prophylactic treatment with LPI did not reduce the 6 year incidence of PACG. . The overall incidence of PACG was $1.61 \%, 95 \%$ confidence interval $(\mathrm{Cl})=1.1-2.25 \%$ which was within the range of estimates predicted at baseline $(0.68 \%-2.10 \%)$. There were more cases of AAC in the control arm compared to screening arm (4 vs. 2, $\mathrm{OR}=2.12,95 \% \mathrm{Cl}=0.39-11.61$ ), which could suggest that a protective effect from prophylactic LPI for incident AAC although the association was also not statistically significant in this small group of cases. Overall there was a low incidence of $\mathrm{AAC}(0.29 \%, 95 \% \mathrm{Cl}=0.11-0.64 \%)$.

No localised lens opacities or increased risk of posterior synechiae was observed in those treated with LPI at baseline, which suggests that LPI is a safe prophylactic treatment. 
236 This was a pragmatic and unique trial in a high risk population. The sample calculations allowed for $23733 \%$ loss to follow up, due to mortality in this older population. The observed death rate in the study

238 population was $17.42 \%(95 \% \mathrm{Cl}=16.33-18.55 \%)$, which was lower than expected, as unrecorded

239 deaths may have occurred.

240 Glaucoma was excluded after randomisation in line with trial protocol. Post randomisation exclusion

241 in trials can introduce selection bias where participants are excluded in a systematic way to favour

242 the intervention. In this trial, fewer participants were excluded with glaucoma in the control group at

243 baseline, and greater numbers of missed glaucoma in the control group would have increased the

244 likelihood of producing a positive result. However, objective criteria for diagnosis of glaucoma were

245 clearly defined before the study and did not involve value judgements. Also, photographs were taken

246 of all optic discs at baseline to determine the effect of missed glaucoma. Grading of baseline

247 photographs suggest that out of 33 PACG cases detected at follow up, 6 may have been missed at

248 baseline, of which 3 were in the screened group and 3 in control. These factors suggest that

249 exclusion bias did not affect the study; furthermore, this trial did not demonstrate an effect, and this

250 type of bias is less pertinent.

251 We were unable to trace $46 \%$ of participants thought to be still alive. This was partly due to

252 unexpected social changes including renumbering dwellings in the outskirts of Ulaanbaatar. We

253 examined differences in baseline characteristics between participants with and without follow up.

254 Traced participants were older and more likely to be male, but there were no differences in screening

255 IOP or CDR at baseline between groups (right CDR: 0.31 without follow up and 0.30 with follow up).

256 As baseline IOP and CDR were associated with incident PACG, these results would suggest that

257 there were no observable clinical differences between groups in ocular parameters. Assuming that

258 there were no differences between groups with and without follow up with respect to primary

259 outcome; then the effect on the trial would be loss of power. With the observed follow up, the

260 effective power for this trial would have been $56 \%$. In order to address the loss to follow up, we used

261 multiple imputation as part of a sensitivity analysis with different imputation models, and the results

262 supported the observations from the complete case analysis. This suggests that a plausible estimate 
was obtained despite the loss to follow up, however, in this instance, it cannot compensate for the missing data.

Sixty nine more participants in the control arm were not followed up. People in the control group who were not screened may be less likely to remember their participation in the study and therefore less likely to return. If there were many cases of PACG in this group, then an effect may have occurred but not detected. However, assuming a high incidence of $2.1 \%$,(based on estimated incidence of $1.4 \%, 95 \% \mathrm{Cl}=0.68-2.1 \%)$, there would only have been an additional $1-2$ cases of glaucoma. $\mathrm{A}$ further 17 cases would be required in the control group to detect a statistically significant difference. It

271 is very unlikely that the incidence of PACG in these 69 participants was nearly $25 \%$, and for a true

272 effect to have been missed due to unequal loss to follow up.

273 The higher incidence of PACG in the intervention group may have occurred by chance. More 274 participants who were not screened received treatment between baseline and follow up; this may have also reduced the incidence of PACG in the control arm. However, excluding all participants with interim LPI, there remains higher incidence of PACG in the screening arm (12 screening arm, 8 in control arm). The intervention under question was both screening with CACD and prevention of PACG with prophylactic LPI treatment. Therefore, we may not have been able to identify an effect because of poor performance of CACD in detection of appropriate cases to treat, or failure of LPI to prevent PACG, or both.

281

282

Only 9 of 19 participants in the intervention group with incident PACG were screen positive at

283 baseline, of which 4 had LPI. Shallow CACD is a risk factor for PAC and PACG. Important risk factors that are important causal factors can perform poorly as screening tests. ${ }^{16}$

Figure 2 shows that there is a large overlap between the distribution of both CACD and screening IOP between participants with incident PACG and those without, which suggests that CACD may not be a good discriminatory test to detect cases that develop incident PACG in this population.

288 Previous studies which have explored screening with ACD include an observational study from 289 Greenland. ${ }^{17}$ Alsbirk identified occludable angles in 20/69 participants based on a cumulative Shaffer 290 grade $<8$, which is less stringent than the present ISGEO criteria. Ten years later, 7 of the 20 (35\%) with occludable angles, and 4 of the $49(8 \%)$ without occludable angles were diagnosed with ISGEO 
equivalent of PAC (relative risk=4.3). More stringent criteria would have classified more progressive cases as non-occludable at baseline. The ISGEO criteria were used in this study, therefore, some participants who may have been at risk of progression did not receive prophylactic treatment.

296 Natural history studies have shown that approximately a quarter of PAC progress to PACG over five

297 years. ${ }^{18}$ Table 4 shows the clinical progression of disease in participants referred for examination. No 298 participants with treated occludable angles progressed to PACG and 6.7\% progressed to PAC over 6 years. This compares favourably to an untreated Indian population of occludable angles where $22 \%$

300 progressed to PAC over 5 years. ${ }^{19}$ Similarly, $11.8 \%$ of participants with PAC progressed to PACG

301 over six years in this study compared to $28.5 \%$ over 5 years in the Indian population. ${ }^{18}$ Although

302 there are differences in population and diagnostic procedures, this comparison suggest that there

303 was a lower rate of disease progression in those treated with LPI,

\section{Conclusions}

305 This trial did not demonstrate a reduction in PACG incidence byscreening with ultrasound

$306 \mathrm{CACD}<2.53 \mathrm{~mm}$ and prophylactic treatment of PAC with LPI. The trial was marred by high loss to

307 follow up. However, this was a pragmatic trial on screening, undertaken in a challenging

308 environment. Screening for angle closure remains a feasible strategy, although newer technologies

309 have been investigated as potential screening tools in this area ${ }^{20}$, and may be more effective than

310 ultrasound CACD. There is some evidence to suggest that LPI is effective in preventing PAC

311 progression. Further work to determine the efficacy of LPI in the prevention of PACG in those with

312 occludable angles and PAC would remove uncertainty about this prophylactic treatment. Such trials

313 are currently underway in East Asia. 


\section{References}

1. Quigley HA, Broman AT. The number of people with glaucoma worldwide in 2010 and 2020 .

Br J Ophthalmol 2006;90:262-7.

2. Foster PJ, Oen FT, Machin D, et al. The prevalence of glaucoma in Chinese residents of Singapore: a cross-sectional population survey of the Tanjong Pagar district. Arch Ophthalmol 2000;118:1105-11.

3. Lowe RF. The natural history and principles of treatment of primary angle-closure glaucoma. Am J Ophthalmol 1966;61:642-51.

4. Lowe RF. Primary angle-closure glaucoma. A review 5 years after bilateral surgery. $\mathrm{Br} \mathrm{J}$ Ophthalmol 1973;57:457-63.

5. Nolan WP, Foster PJ, Devereux JG, Uranchimeg D, Johnson GJ, Baasanhu J. YAG laser iridotomy treatment for primary angle closure in east Asian eyes. $\mathrm{Br} J$ Ophthalmol 2000;84:1255-9.

6. Alsbirk PH. Anterior chamber depth and primary angle-closure glaucoma. I. An epidemiologic study in Greenland Eskimos. Acta Ophthalmol (Copenh) 1975;53:89-104.

7. Foster PJ, Alsbirk PH, Baasanhu J, Munkhbayar D, Uranchimeg D, Johnson GJ. Anterior chamber depth in Mongolians: variation with age, sex, and method of measurement. Am J Ophthalmol 1997;124:53-60.

8. Wilson J, Jungner G. Principles and Practice of Screening for Disease. Geneva: World Health Organization, 1968.

9. Nolan WP, Baasanhu J, Undraa A, Uranchimeg D, Ganzorig S, Johnson GJ. Screening for primary angle closure in Mongolia: a randomised controlled trial to determine whether screening and prophylactic treatment will reduce the incidence of primary angle closure glaucoma in an east Asian population. Br J Ophthalmol 2003;87:271-274.

10. Foster PJ, Buhrmann R, Quigley HA, Johnson GJ. The definition and classification of glaucoma in prevalence surveys. Br J Ophthalmol 2002;86:238-42.

11. Devereux JG, Foster PJ, Baasanhu J, et al. Anterior chamber depth measurement as a screening tool for primary angle-closure glaucoma in an East Asian population. Arch Ophthalmol 2000;118:257-63.

12. Ocular Hypertension Treatment Study (OHTS II). Manual of Procedures. version 4.0, 2003. 
13. Schafer J, Olsen M. Analysis of incomplete multivariate data. London: Chapman and Hall, 1998.

14. Royston P. Multiple Imputation. Stata Journal 2004;4.

15. Royston P. Multiple Imputation of Missing values: further update of ice, with emphasis on interval censoring. Stata Journal 2007;7.

16. Wald NJ, Hackshaw AK, Frost CD. When can a risk factor be used as a worthwhile screening test? BMJ 1999;319:1562-5.

17. Alsbirk $\mathrm{PH}$. Anatomical risk factors in primary angle-closure glaucoma. A ten year follow up survey based on limbal and axial anterior chamber depths in a high risk population. Int Ophthalmol 1992;16:265-72.

18. Thomas R, Parikh R, Muliyil J, Kumar RS. Five-year risk of progression of primary angle closure to primary angle closure glaucoma: a population-based study. Acta Ophthalmol Scand 2003;81:480-5.

19. Thomas R, George R, Parikh R, Muliyil J, Jacob A. Five year risk of progression of primary angle closure suspects to primary angle closure: a population based study. Br J Ophthalmol $2003 ; 87: 450-4$

20. Nolan WP, Aung T, Machin D, et al. Detection of Narrow Angles and Established Angle Closure In Chinese Residents of Singapore: Potential Screening Tests. Am J Ophthalmol 2006.

\section{Table 1: Criteria for visual field tests at follow up}

Disc suspects: Eyes with a CDR or CDR asymmetry $\geq 97.5^{\text {th }}$ percentile for the normal population (0.7 CDR and 0.2 asymmetry for Mongolians)

Those with optic disc margin haemorrhages

Those with an IOP $\geq 97.5^{\text {th }}$ percentile $(19 \mathrm{mmHg}$ for Mongolians)

Those with an occludable drainage angle, but normal optic disc, IOP and no PAS

$\mathrm{CDR}=$ cup disc ratio $\mathrm{IOP}=$ intraocular pressure $\mathrm{PAS}=$ peripheral anterior synechiae 
Figure 1. Flow chart of participants in the trial. All participants were Mongolian volunteers. Glaucoma was excluded in all randomised participants prior to receiving screening or no screening using direct ophthalmoscopy and slitlamp examination+/- visual field assessment where indicated. 32 participants from the control arm were examined due to observations on direct ophthalmoscopy. All clinical diagnoses of primary angle closure were based on ISGEO definitions.

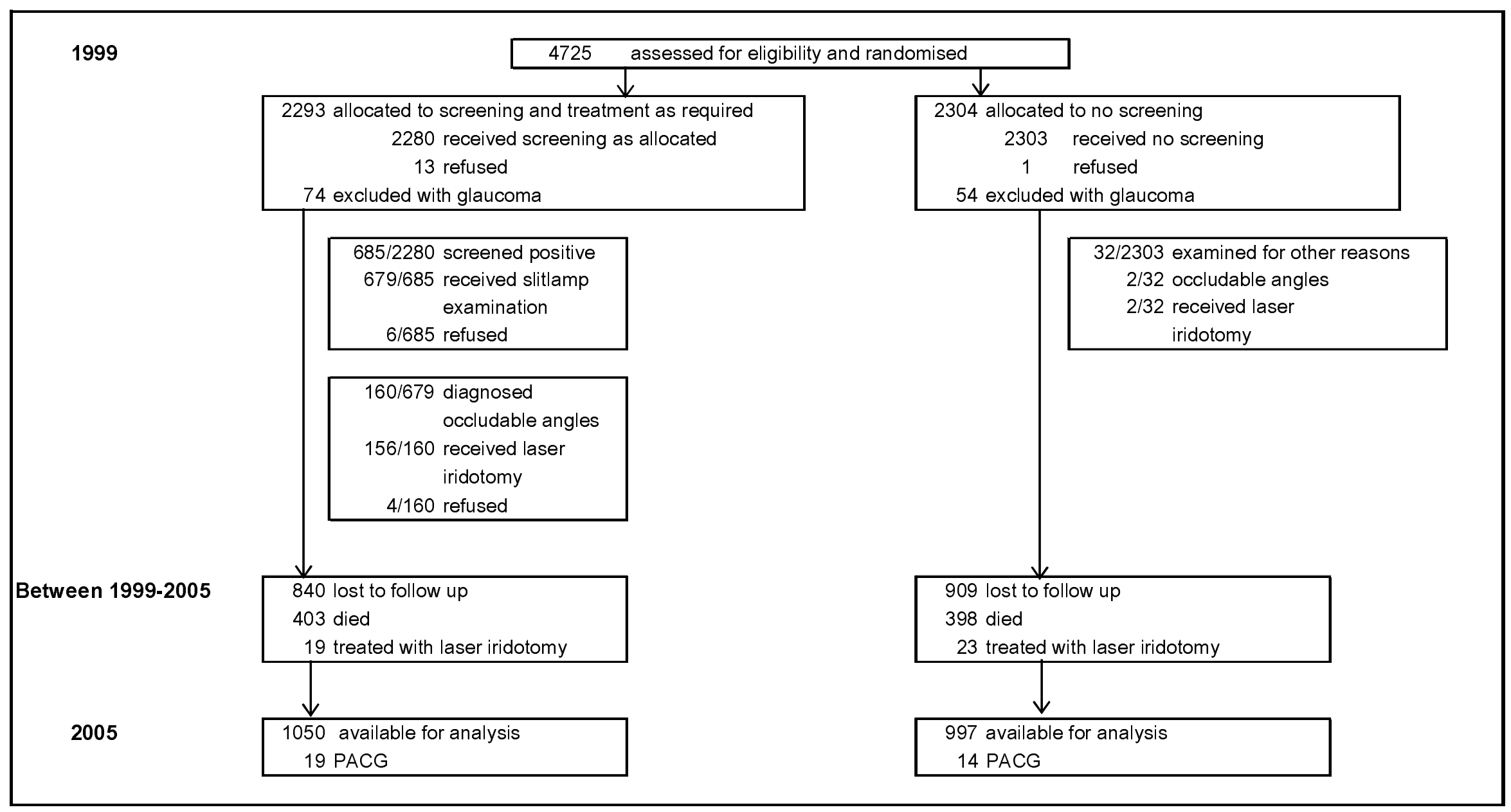


Table 2. Incident cases of Primary angle closure glaucoma as identified by different methods.

\begin{tabular}{|c|c|c|c|c|c|c|c|c|c|c|c|c|c|c|}
\hline \multirow{3}{*}{$\begin{array}{l}\text { Type of } \\
\text { Evidence }\end{array}$} & \multirow[t]{3}{*}{ Method of Identification } & \multicolumn{2}{|c|}{ Right Eye } & \multicolumn{2}{|c|}{ Left Eye } & \multicolumn{2}{|c|}{ Either Eye } & \multicolumn{2}{|r|}{ Either } & \multicolumn{2}{|l|}{ Eye } & & & \multirow{3}{*}{ p-value ${ }^{+}$} \\
\hline & & & & & & & & \multicolumn{2}{|c|}{$\begin{array}{c}\text { Group A } \\
\text { (intervention) }\end{array}$} & \multicolumn{2}{|c|}{$\begin{array}{l}\text { Group B } \\
\text { (control) }\end{array}$} & \multirow{2}{*}{\multicolumn{2}{|c|}{$\begin{array}{l}\text { Risk Ratio } \\
\text { N }(95 \% \mathrm{Cl})\end{array}$}} & \\
\hline & & \multicolumn{2}{|c|}{$\mathrm{N}(\%)$} & \multicolumn{2}{|c|}{$\mathrm{N}(\%)$} & \multicolumn{2}{|c|}{$\mathrm{N}(\%)$} & $n$ & $(\%)$ & $n$ & $(\%)$ & & & \\
\hline \multirow{4}{*}{$\begin{array}{l}\text { Structural } \\
\text { and } \\
\text { Functional } \\
\text { Evidence }\end{array}$} & $\begin{array}{l}\text { Both Clinical examination } \\
\text { and photographic grading } \\
\text { with Visual field grading }\end{array}$ & & $(0.05)$ & 0 & (0) & 1 & $(0.05)$ & 0 & (0) & 1 & $(0.10)$ & & & \\
\hline & $\begin{array}{l}\text { Clinical Examination with } \\
\text { Visual field grading }\end{array}$ & 14 & $(0.68)$ & 9 & $(0.44)$ & 18 & $(0.88)$ & 11 & $(1.05)$ & 7 & $(0.70)$ & & & \\
\hline & $\begin{array}{l}\text { Photographic grading with } \\
\text { Visual Field Grading }\end{array}$ & 0 & (0) & 0 & (0) & 0 & (0) & 0 & (0) & 0 & (0) & & & \\
\hline & $\begin{array}{l}\text { All Structural and } \\
\text { Functional evidence }\end{array}$ & 15 & $(0.73)$ & 9 & $(0.44)$ & $19^{*}$ & $(0.93)$ & 11 & $(1.05)$ & 8 & $(0.80)$ & 1.31 & $(0.52-3.23)$ & 0.56 \\
\hline \multirow{4}{*}{$\begin{array}{l}\text { Structural } \\
\text { Evidence } \\
\text { only }\end{array}$} & $\begin{array}{l}\text { Both Clinical examination } \\
\text { and photographic grading }\end{array}$ & 2 & $(0.10)$ & 4 & $(0.20)$ & 5 & $(0.24)$ & 2 & $(0.19)$ & 3 & $(0.30)$ & & & \\
\hline & Clinical examination & 7 & $(0.34)$ & 6 & $(0.29)$ & 8 & $(0.39)$ & 5 & $(0.48)$ & 3 & $(0.30)$ & & & \\
\hline & Photographic Grading & 1 & $(0.05)$ & 2 & $(0.10)$ & 1 & $(0.05)$ & 1 & $(0.10)$ & 0 & (0) & & & \\
\hline & All Structural Evidence & 10 & $(0.49)$ & 12 & $(0.59)$ & 14 & $(0.68)$ & 8 & $(0.76)$ & 6 & $(0.60)$ & 1.27 & $(0.44-3.64)$ & 0.66 \\
\hline Overall & $\begin{array}{l}\text { All types of evidence } \\
\text { (observed data) }\end{array}$ & 25 & $(1.22)$ & 21 & $(1.03)$ & 33 & $(1.61)$ & 19 & $(1.81)$ & 14 & $(1.40)$ & 1.29 & $(0.65-2.60)$ & 0.47 \\
\hline $\mathbf{N}$ & $\begin{array}{l}\text { Total examined during } \\
\text { follow up }\end{array}$ & & 2047 & & 47 & & 47 & & & & 997 & & & \\
\hline
\end{tabular}

"Of 19 cases with both structural and functional evidence, 17 cases had 2 or more visual fields and 2 cases only had 1 visual field.

+Pearson's $x^{2}$ test

Only participants with no evidence of secondary causes of glaucoma in the affected eye and evidence of primary angle closure using ISGEO definitions were categorised as case. 
Table 3. Diagnoses of participants treated with peripheral iridotomy between baseline and follow up studies

Screened Group

n (\%)

$4(21.1)$

7 (36.8)

Presumed previous occludable angles

(open angles on follow up)

Total

Acute angle closure

2

(final diagnosis=PACG)
7 (36.8)

1 (5.3)

Control Group

n (\%)

19

23

4

(final diagnosis,

2=PACG, 2=PAC)

$6(26.1)$

$4(17.4)$

4 (17.4)

$9(39.2)$ 
Figure 2. Distribution of $C A C D$ and IOP in participants with incident PACG and those with no PACG at follow up. The CACD readings were calculated from the mean of three slitlamp-mounted ultrasound measurements of CACD. The IOP readings were obtained from Tonopen IOP readings. All measurements were from the right eye only.
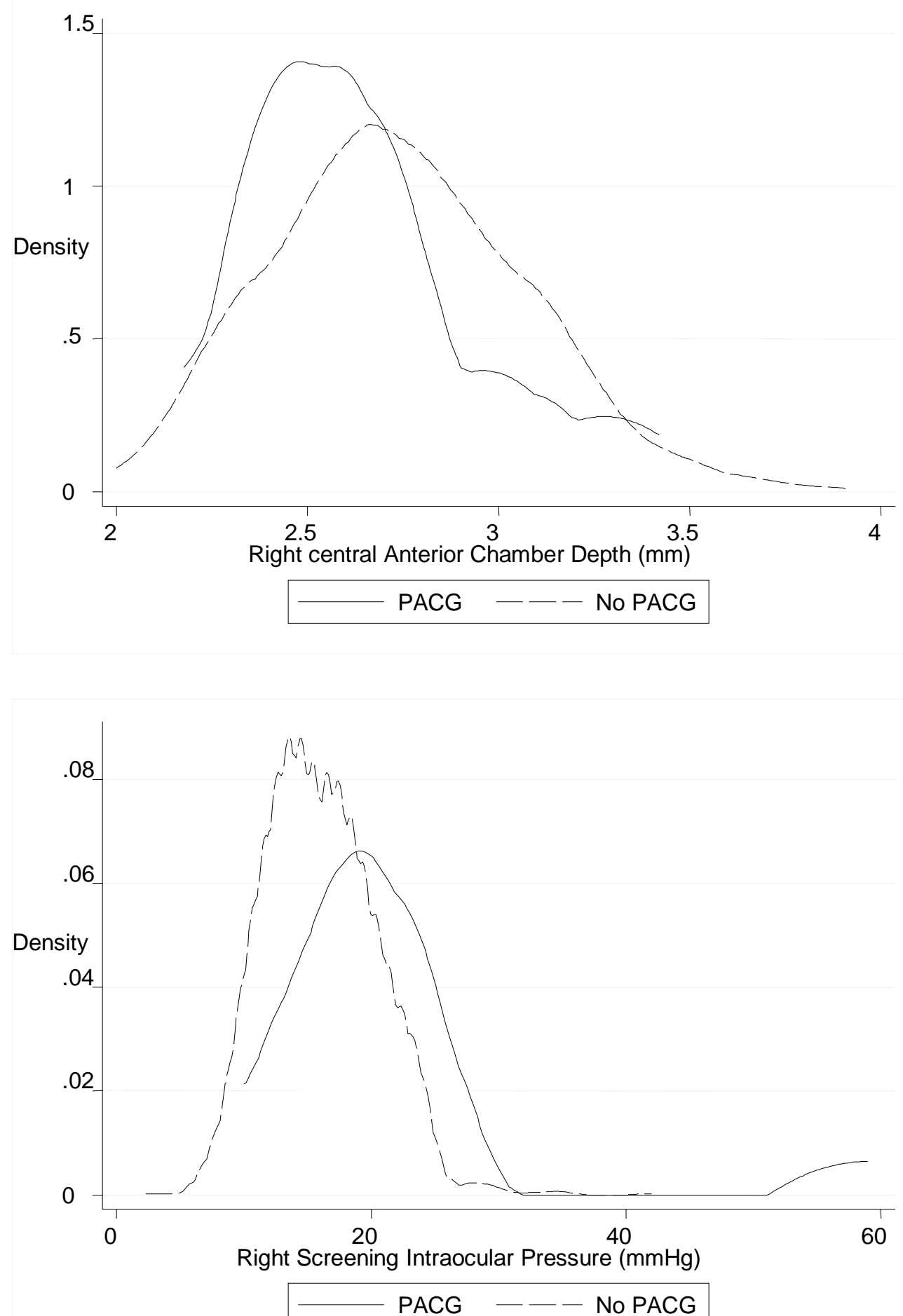

$\mathrm{CACD}=$ central anterior chamber depth $\mathrm{IOP}=$ intraocular pressure $\mathrm{PACG}=$ primary angle closure glaucoma 
Table 4. Clinical Progression of Disease from baseline diagnosis.

\section{Baseline Diagnosis}

\begin{tabular}{|c|c|c|c|c|c|c|c|}
\hline & & \multicolumn{2}{|c|}{ Normal } & \multicolumn{2}{|c|}{ Occludable angles } & \multicolumn{2}{|c|}{ PAC } \\
\hline & Normal & 157 & $(66.5)$ & 32 & $(71.1)$ & $13^{*}$ & $(38.2)$ \\
\hline Follow up & Occludable & 38 & $(16.1)$ & 5 & (11.1) & $2^{+}$ & $(5.9)$ \\
\hline \multirow[t]{5}{*}{ Diagnosis } & angles & & & & & & \\
\hline & PAC & 9 & (3.8) & 3 & $(6.7)$ & 10 & $(29.4)$ \\
\hline & PACG & 6 & $(2.7)$ & 0 & $(0)$ & 4 & $(11.8)$ \\
\hline & Other ${ }^{\S}$ & 26 & $(10.6)$ & 5 & $(11.0)$ & 5 & $(14.6)$ \\
\hline & Total & 23 & & & 45 & & 34 \\
\hline
\end{tabular}

PAC=primary angle closure PACG=primary angle closure glaucoma PAS=peripheral anterior synechia

All participants in this group with occludable angles and PAC at baseline were treated with LPI at baseline.

*12 participants had PAS at baseline, 7/12 were also diagnosed with PAS at followup, but with open angles.

${ }^{+}$None of these participants had PAS at baseline

§Other diagnoses included secondary glaucoma, open angle glaucoma, ocular hypertension and glaucoma suspects with open angles. 


\section{Acknowledgements}

We would like to thank Dr Davaasambuu Tsendenkhuu, Chimed Oyunsuren, Tsedengonbo

Lhagvasuren, Dr Altsentseg from Suhkbaatar and Bayanzurkh hospitals, the staff of Suhkbaatar

district hospital, Bayanhongor hospital and Bolor Melmii who worked on this project. We are also grateful to Dr lan White for advice on statistical analysis. 


\section{Appendix}

\section{Multiple Imputation (MI) Procedure}

Analyses for primary outcome were performed on observed data only. We used multiple imputation to estimate the potential effect of the missing data and effective loss of power in a sensitivity analysis. The multiple imputation procedure substitutes missing values with several versions of imputed values. There is an underlying assumption that missing data is missing at random, where the absence is not dependent on unobserved variables.

Logistic regression models were fitted to the imputed data.

Imputation

A logistic regression model was fitted using available baseline predictors of primary outcome, including age, sex, allocation group, right and left cup disc ratio(CDR), right and left central anterior chamber depth and diagnosis at baseline. The follow up variables, primary outcome, presence of an occludable angle, right and left CDR and death were also included in different models. Separate models were also generated to incorporate interactions between diagnosis and age. As not all subjects had diagnosis at baseline, this was also imputed using logistic regression using age, sex, right CDR and left CDR as predictors after conversion to a binary variable (occludable or open angle). Variables were chosen based on association with "missingness", relevance to final analysis, and known association with outcome.

Assumptions of normality were dealt with by using the "match" option, or by bootstrap sampling. Fifty sets of imputed values for primary outcome were drawn from their distributions, conditional upon the observed data, and subsequently merged with the observed values to create fifty imputed data sets. Fifty sets were selected due to the relatively high proportion of missing values.

\section{Analysis}

Logistic regression analyses were performed on the imputed datasets. The resulting estimates and confidence intervals were noted. The parameter estimate from the complete case analysis (risk ratio $=1.29)$ was compared to the range of estimates from the imputed dataset analyses. 